\title{
A NEW METHOD FOR CALCULATING THE \\ FLOW FIELD IN A DUMP COMBUSTOR,
}

by

$$
\text { Alan A. Hale }
$$

Thesis submitted to the Graduate Faculty of the Virginia Polytechnic Institute and State University in partial fulfillment of requirements for the degree of

MASTER OF SCIENCE

in

Mechanical Engineering

APPROVED :

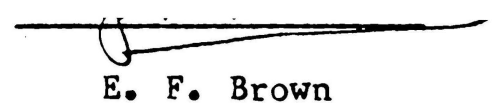

F. J. Plerce

S. B. Thomason.

June, 1984

Blacksburg, Virginia 
A NEW METHOD FOR CALCULATING THE

FLOW FIELD IN A DUMP COMBUSTOR

by

Alan A. Hale

(ABSTRACT)

An elliptic/parabolic procedure for solving the flow field in a dump combustor is described. The elliptic calculation is used at the dump combustor entrance and a parabolic calculation downstream. The calculations are restricted to steady axisymmetric nonreactive incompressible flow. A great savings in computation time and computer storage is obtalned with no observable compromise in accuracy. 


\section{ACKNOWLEDGEMENTS}

This work was made possible by a research contract with the Air Force Office of Scientific Research. I am grateful to

for typing this thesis. I am also grateful to

for her sincere concern and willingness to share her experiences in the numerical flow field calculations. I am especially grateful for the encouragement and advice from my adviser Dr. Eugene F. Brown which resulted in the successful completion of this work. 
TABLE OF CONTENTS

Page

ABSTRACT $\ldots \ldots \ldots \ldots \ldots \ldots \ldots \ldots \ldots \ldots \ldots \ldots \ldots \ldots \ldots \ldots \ldots \ldots \ldots \ldots \ldots \ldots \ldots \ldots \ldots \ldots \ldots 11$

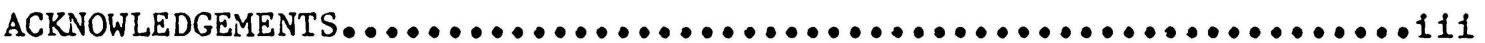

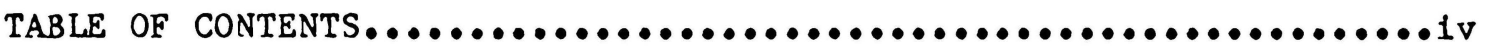

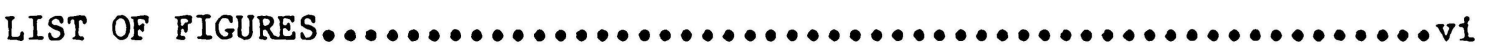

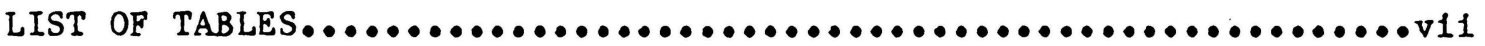

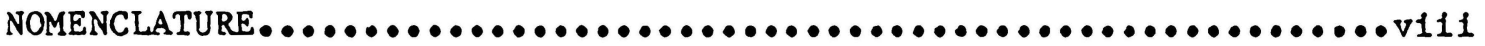

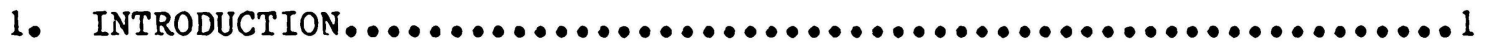

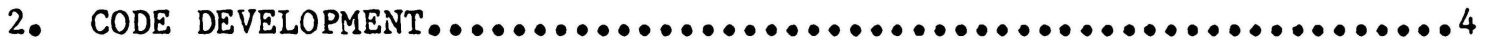

2.1 Differential Equations..............................4

2.2 Finite Difference Equations..........................7

2.3 Boundary Conditions...............................13

2.4 Solution Technique.................................17

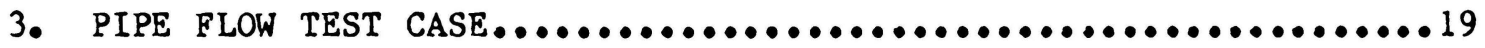

3.1 Problem Description...............................19

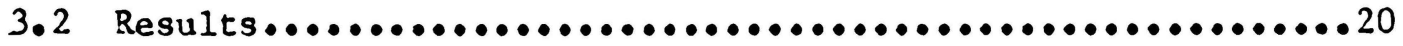

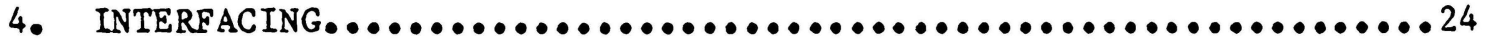

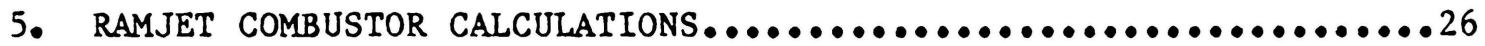

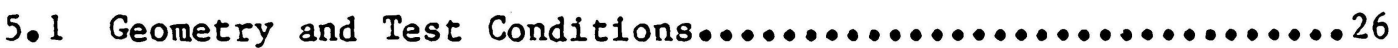

5.2 Calculations $\ldots \ldots \ldots \ldots \ldots \ldots \ldots \ldots \ldots \ldots \ldots \ldots \ldots \ldots \ldots \ldots \ldots \ldots \ldots \ldots \ldots \ldots \ldots \ldots \ldots .26$

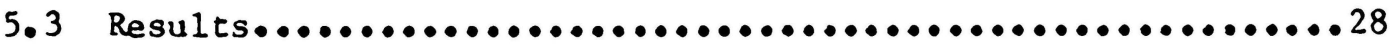

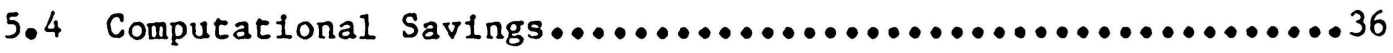

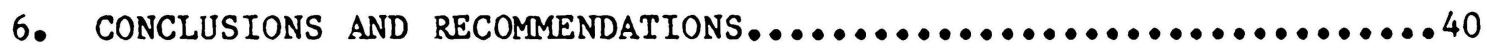

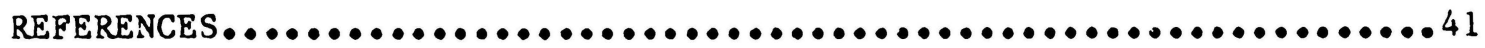


TABLE OF CONTENTS (continued)

Page

APPENDIX A...........................................43

APPENDIX B...........................................46

APPENDIX C.............................................48

APPENDIX D...............................................51

VITA $\quad$ V...............................................54 


\section{LIST OF FIGURES}

Figure

Title

Page

1 Interior Elliptic Control Volume.....................5

2 Interior Parabolic Control Volume......................9

3 Wall Parabolic Control Volume......................15

4 Axial Velocity Profile Calculations for Fully

Developed Pipe Flow...................................21

5 Dump Combustor Axial Grid Spacing Used for the

Elliptic Method (A) and the Hybrid Method (B)............27

6 Axial Velocity Results for the Hybrid Method,

Elliptic Method, and Experimental Data at Station 40......29

7 Turbulent Intensity Results for the Hybrid Method,

Elliptic Method, and Experimental Data at Station 40.......30

8 Axial Velocity Results for the Hybrid Method, Elliptic Method, and Experimental Data at Station 43........33

9 Turbulent Intensity Results for the Hybrid Method, Elliptic Method, and Experimental Data at Station 43.......34

10 Centerline Turbulent Intensity for Elliptic

Calculations and Experimental Data at Each x station.•.....35

11 Radial Velocity at Every Twentieth $\Delta x$ From Station

35 to Station 43 by the Hybrid Method With a Step

Size of 0.001 m....................................37 


\section{LIST OF TABLES}

Table

Title

Page

I Diffusion Coefficients and Source Terms $\left(s^{\phi} \bar{r} \Delta r \Delta x=s_{1}^{\phi} \phi_{\mathrm{p}}+s_{2}^{\phi}\right)$

II Axtal Pressure Gradient Calculations at Every Tenth

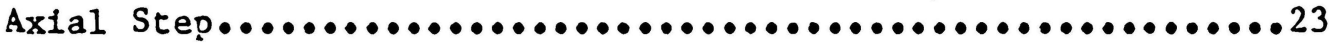


NOMENCLATURE
$A, B$
Coefficient in finite difference equations
$C_{\mu}, C_{D}, C_{1}, C_{2}$
Turbulent modeling coefficients
D
Coefficient of the pressure gradient term
E
Integration constant
G
Source term for turbulent modeling, defined in Table 1
$\mathrm{K}$
Turbulence kinetic energy
L
Length of dump combustor
m
Net inflow of mass
$\overline{\mathrm{p}}$
Pressure in the axial momentum equation
p
Pressure in the radial direction
$\operatorname{Re}$
Reynolds number based on pipe diameter
r
Radial coordinate
$\overline{\mathbf{r}}$
Radial distance from the centerline to the center of a control volume
$R_{1} \quad$ Radius of the dump combustor
$\mathrm{R}_{2}$
Radius before sudden expansion
S
Source term, defined in Table 1
$\mathrm{s}_{1}, \mathrm{~s}_{2}$
Coefficients for the linearlzed source term, defined in Table 1
T Temperature
TI Turbulent intensity
U
Axial velocity before expansion
$\mathrm{u}_{\tau}$
Friction velocity, defined in Appendix $A$
$u^{+}$
Axial velocity nondimensionalized by friction velocity 
NOMENCLATURE (continued)

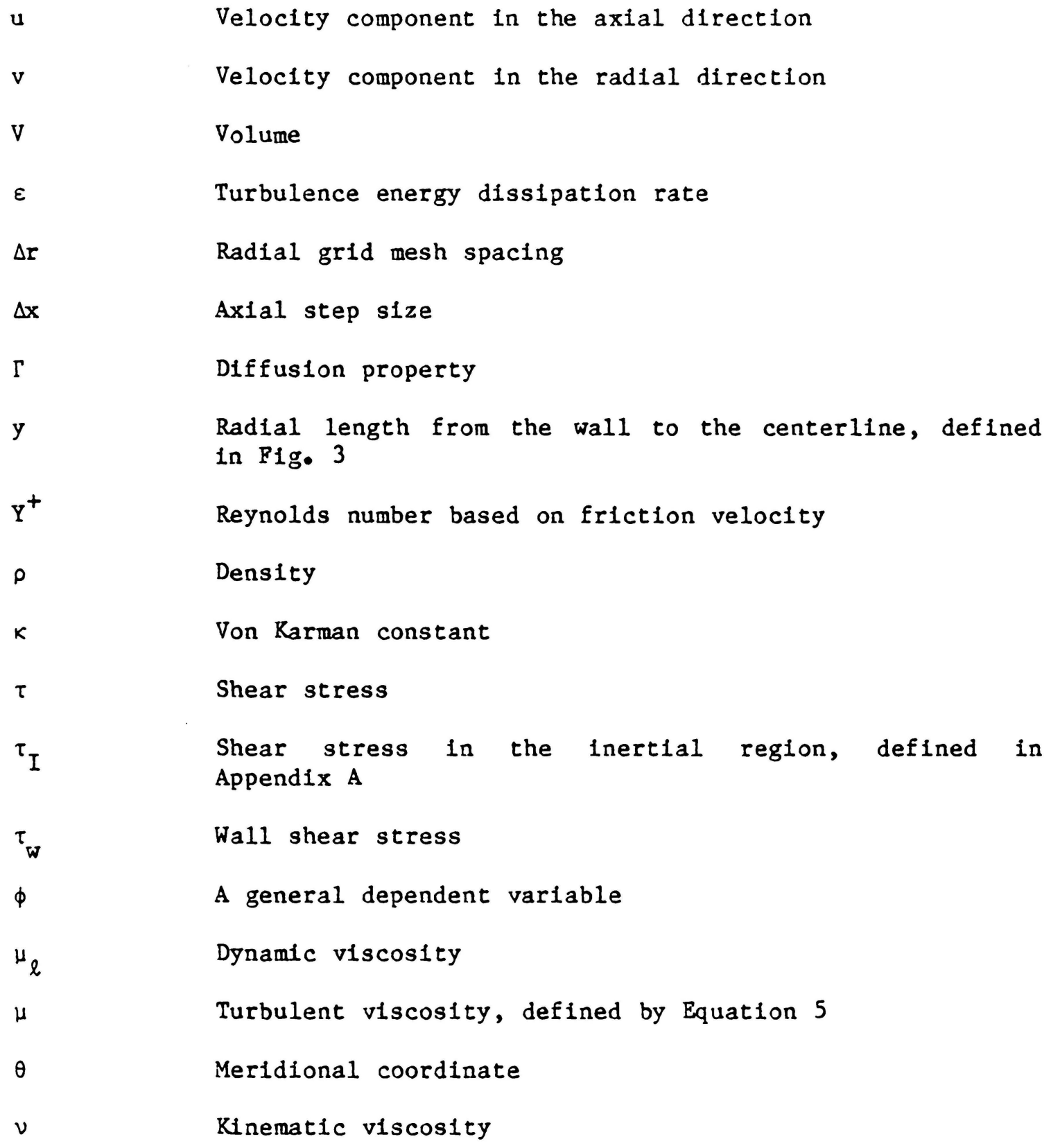

Superscripts

$\mathrm{p}, \mathrm{u}, \mathrm{v}, \mathrm{k}, \varepsilon \quad$ Corresponding dependent variables

$\phi \quad$ General dependent variable 
NOMENCLATURE (continued)

$\begin{array}{ll}* & \text { Estimated pressure } \\ \vdots & \text { Correction term } \\ & \text { Source term in differential equations }\end{array}$

Subscripts

Centerline

D Downstream station

$\max$ Maximum over the pipe cross section

E,W Corresponding points, defined in Figure 1

$\mathrm{N}, \mathrm{n}, \mathrm{P}, \mathrm{s}, \mathrm{S} \quad$ Corresponding points, defined in Figure 2

$\phi \quad$ General dependent variable

U Upstream station 


\section{INTRODUCTION}

\subsection{Problem Description}

A dump combustor is a sudden expansion which makes use of the sudden increase in area to produce a flame holding effect. The flow in dump combustors has been solved by a number of investigators over the past few years. Examples of these solutions can be found in Gosman et al. (1), Lilley and Rhode (2), Novick et al• (3), and Syed and Sturgess (4). A common characteristic of these solutions is the mathematical modeling of the flow using the Navier-Stokes equations.

The flow in a dump combustor may be viewed as having two distinct regions with very different characteristics. The first region, which starts at the sudden expansion and extends to the point of reattachment, is the recirculation region. Because of the presence of the separation region the pressure-field exerts a strong upstream Influence and therefore the flow is said to be elliptic.

The second region, after reattachment, is the plpe flow region. In this region the flow is "one-way" (5) meaning:

a) there is no backflow in the main direction of flow,

b) the streamwise diffusion of energy, momentum, and mass is negligible, and

c) the upstream flow conditions are unaffected by a downstream pressure fleld.

In this region the elliptic effect of the Navier-Stokes equations disappears and the flow becomes parabolic in nature. The purpose of this thesis is to describe a new computational method for dump 
combustor calculations which takes advantage of this characteristic. In particular it is proposed to replace the solution of the NavierStokes equations with the solution of the parabolized Navier-Stokes equations in this region. It will be shown that this results in a considerable savings of computational time and computer storage. References involving solutions of the parabolized Navier-Stokes equations are numerous and can be found, for example, in Patankar and Spalding $(5,6)$ and Spalding (7).

The solution technique for the elliptic and parabolized NavierStokes equations are very different. The elliptic method is implicit so each dependent variable calculated in the flow fleld will have a value stored at each computational node. A line relaxation technique is used to calculate the flow field iteratively until the flow field calculated is within a desired tolerance. A disadvantage of using the Navier-Stokes equations is the large computational time required to carry out these iterations. Often a grid that expands in the axial directon is used to decrease not only the computational time but the computer storage. Sometimes the consequence of using this coarser $x-$ grid mesh is the presence of a serious decrease in accuracy•

The parabolized Navier-Stokes equations are obtained from the Navier-Stokes equations by neglecting the streamwise diffusion terms. This allows the equations to be solved by a streamwise marching integration procedure. Clearly only a small amount of computer storage is needed. Since the flow field is not obtained by iteration the computation time is short. 
The combined elliptic and parabolic procedure just described I call the hybrid method. The purpose of this thesis will be to discuss how this hybrid method was developed, to discuss the interfacing which must take place between the two methods of solution, and to compare the results of the hybrid solution with the elliptic calculations and experimental data. 


\section{CODE DEVELOPMENT}

An elliptic code called STARPIC, written by Lilley and Rhode (2), is used to calculate the flow fleld in the entrance of the dump combustor. This elliptic code solves the Navier-Stokes equations on a staggered grid control volume shown in Figure 1. The TEACH code and SIMPLE algorithm described in Patankar (8) are used. This program was made avallable to me by the Ramjet Technology Branch, Aero Propulsion Laboratory, at the Wright-Patterson Alr Force Base. A parabolic code, however, was not avallable, therefore a considerable amount of time was spent developing such a code patterned after STARPIC. This section will be mostly concerned with the development of this code. The calculations are restricted to incompressible, axisymmetric, steady flow.

\subsection{Differential Equations}

The set of governing equations for the parabolic method are conservation of mass, the axlal Navier-Stokes equation (axial momentum equation), the transport equation for turbulent kinetic energy, and the transport equation for turbulent dissipation rate. These equations are presented below in cylindrical coordinates (5). 


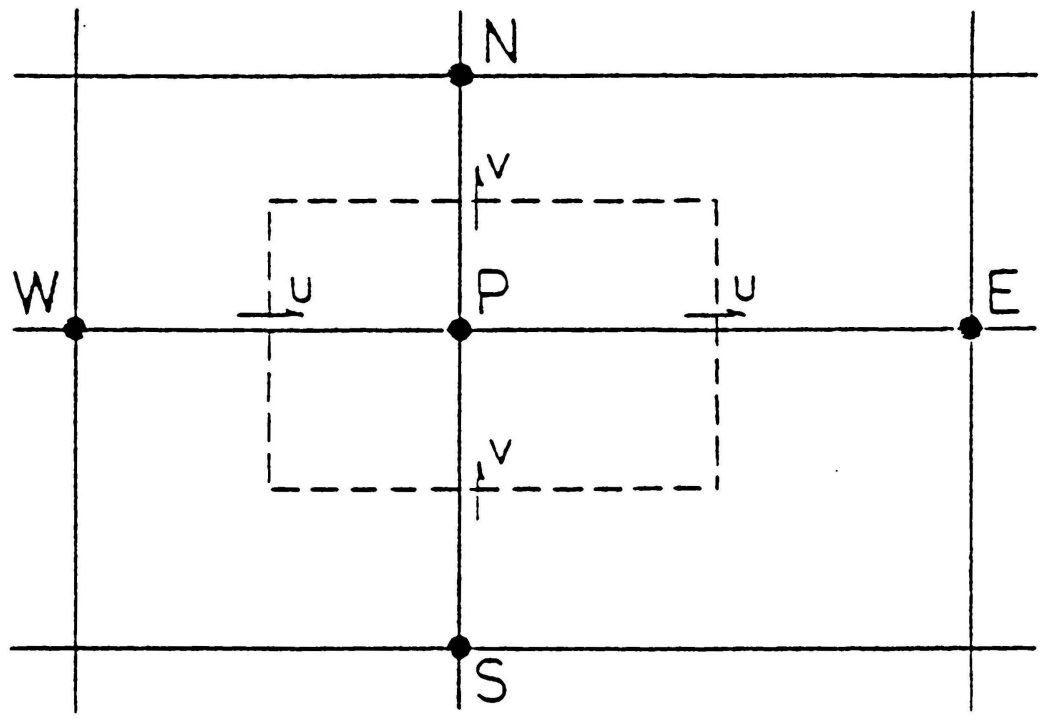

Fig. 1. Interior Elliptic Control Volume 
Continuity:

$$
\frac{1}{r}\left(\frac{\partial}{\partial x}(r \rho u)+\frac{\partial}{\partial r}(r \rho v)\right)=0
$$

Axial Momentum:

$$
\frac{1}{r}\left[\frac{\partial}{\partial x}(r \rho u u)+\frac{\partial}{\partial r}(r \rho v u)-\frac{\partial}{\partial r}\left(r \Gamma^{u} \frac{\partial u}{\partial r}\right) \approx=-\frac{\partial \bar{P}}{\partial x}+\hat{S}^{u}\right.
$$

Turbulent Kinetic Energy, k:

$$
\frac{1}{r}\left[\frac{\partial}{\partial x}(r \rho u k)+\frac{\partial}{\partial r}(r \rho v k)-\frac{\partial}{\partial r}\left(r \Gamma^{k} \frac{\partial k}{\partial r}\right)=\hat{S}^{k}\right.
$$

Turbulent Dissipation Energy Rate, $\varepsilon$ :

$$
\frac{1}{r}\left[\frac{\partial}{\partial x}(r \rho u \varepsilon)+\frac{\partial}{\partial r}(r \rho v \varepsilon)-\frac{\partial}{\partial r}\left(r \Gamma^{\varepsilon} \frac{\partial \varepsilon}{\partial r}\right) \sim=\hat{s}^{\varepsilon}\right.
$$

The parabolized computational procedure means that the diffusion terms

$$
\frac{1}{r} \frac{\partial}{\partial x}\left(r \Gamma^{u} \frac{\partial u}{\partial x}\right) ; \quad \frac{1}{r} \frac{\partial}{\partial x}\left(r \Gamma^{k} \frac{\partial k}{\partial x}\right) ; \quad \text { and } \frac{1}{r} \frac{\partial}{\partial x}\left(r \Gamma \frac{\partial \varepsilon}{\partial x}\right)
$$

normally present if the elliptic calculations had been carried out have been eliminated from the above equations. The rational for eliminating these terms is that they present a mechanism by which upstream diffusion may take place which is contrary to the definition of a parabolic flow. The $u, k$, and $\varepsilon$ superscripts refer to specific terms unique to the axial momentum, turbulence kinetic energy, and 
turbulence dissipation rate equations respectively• The $\Gamma^{u}, \Gamma^{k}$, and $\Gamma^{\varepsilon}$ represent the diffusion coefficients of axial momentum, turbulence kinetic energy, and turbulence dissipation rate respectively. The diffusion coefficients and the linearized source terms appearing in these equations will be defined once the finite difference form of these equations has been developed.

\subsection{Finite Difference Equations}

The finite difference equations for the parabollc method use control volumes based on a semi-staggered grid system 11lustrated in Figure 2. The radial velocity $(v)$ is calculated midway between nodes $S$ and $P$ but stored on node $P$. The axial velocity (u), like all other dependent variables, is stored on node $P$. The semi-staggered grid improves and simplifies the convection term calculation by calculating the velocities at the center of each control face. where needed. The governing equations of the parabolic method (1-4) may be written in a form such that $\phi$ represents any property which can be convected or diffused (5), giving:

$$
\frac{1}{r}\left[\frac{\partial}{\partial x}(\rho u r \phi)+\frac{\partial}{\partial r}(\rho \operatorname{vr} \phi)-\frac{\partial}{\partial r}\left(r \Gamma \phi \frac{\partial \phi}{\partial r}\right)\right]=\widehat{S}^{\phi}
$$

From this equation $\phi$ may take on the values $1, u, k$, and $\varepsilon$ thereby recovering the conservation of mass, axial momentum, turbulence kinetic energy, and turbulence dissipation rate equations respectively. A general differential equation in $\phi$ is conventent for presenting the finite difference equations.

The development of the finite difference equations make 


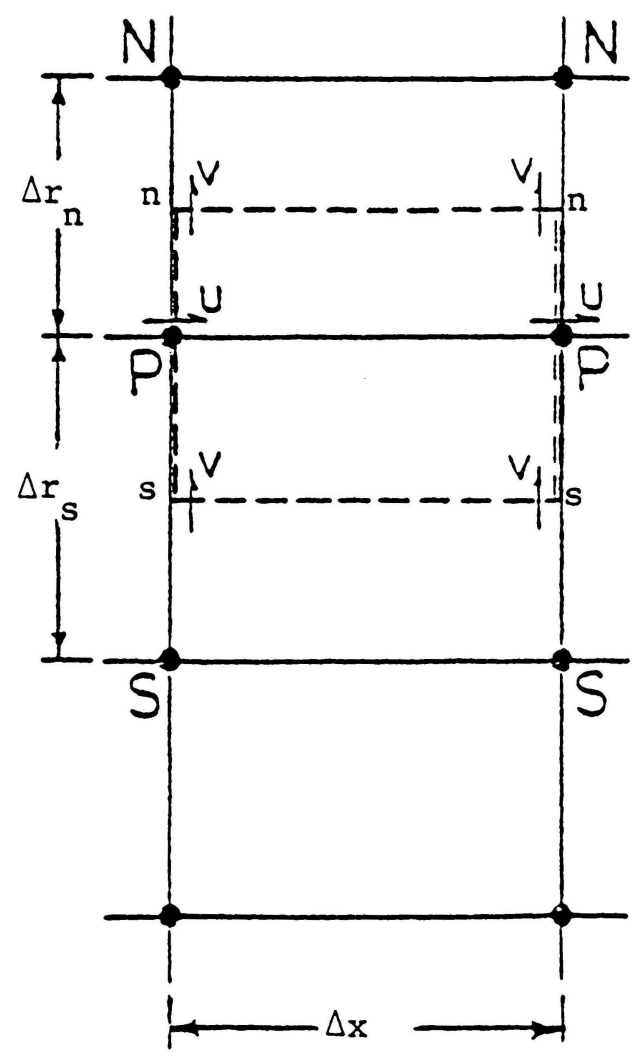

Fig. 2. Interior Parabolic Control Volume 
assumptions on the change of $\phi$ between grid points (5).

a) In the axial direction $\phi$ is a stepwise property $\phi=\phi_{D}$ at the downstream face of a control volume up to, but not including, the upstream face of the same control volume.

b) The upstream and downstream face of each control volume falls on the $r \theta$ plane. The $\phi$ associated with an $r \theta$ face of a control volume is stepwise. This means $\phi_{p}$ remains fixed on the face of the control volume but suddenly changes to $\phi_{\mathrm{N}}$ or $\phi_{\mathrm{S}}$ when exiting radially to an adjacent control volume.

c) The radial top and bottom face of each control volume is an $x \theta$ surface. The numerical value of $\phi$ convected on a radial control volume face will be the arithmetic mean of the adjacent radial $\phi$ values.

d) In the diffusion of $\phi$ across the same $x \theta$ surface of a control volume a linear profile in $\phi$ between adjacent radial $\phi$ values will be assumed.

The finite difference equations based on the above assumptions are developed by integrating Equation 7 over the control volume, giving: 


$$
\begin{gathered}
(\bar{r} \Delta r \rho u)_{P, D} \phi_{P, D}-(\bar{r} \Delta r \rho u)_{P, U} \phi_{P, U} \\
+\left(r_{n} \Delta x \rho v\right)_{n, U}\left(\frac{\phi_{N, D}+\phi_{P, D}}{2}\right)-\left(r_{s} \Delta x \rho v\right)_{s, U}\left(\frac{\phi_{P, D}+\phi_{S, D}}{2}\right) \\
=\left(r_{n} \Delta x \Gamma^{\phi}\right)_{n, U}\left(\frac{\phi_{N, D}-\phi_{P, D}}{\Delta Y_{n}}\right)-\left(r_{s} \Delta x \Gamma^{\phi}\right)_{S, U}\left(\frac{\phi_{P, D}-\phi_{s, D}}{\Delta Y_{s}}\right) \\
+s^{\phi} \bar{r} \Delta r \Delta x
\end{gathered}
$$

See Table I for one definition of the diffusion coefficients, $\Gamma^{\phi}$, and source term $S^{\phi}$. The coefficient $(\bar{r} \Delta r \rho u)_{P, D}$ multiplied by $\phi_{P, D}$ in the axial convection term must be rewritten to eliminate the unknown downstream influence on axial velocity (5). To eliminate this problem the continuity equation is written in fintte difference form and integrated over the control volume. The continulty equation is solved for the troublesome coefficient just discussed.

$$
(\bar{r} \Delta r \rho u)_{P, D}=(\bar{r} \Delta r \rho u)_{P, U}-\left(r_{n} \Delta x \rho v\right)_{n, U}+\left(r_{s} \Delta x \rho v\right)_{s, U}
$$

Making this substitution and rearranging the terms in the general $\phi$ finte difference equation produces the following general discretization equations.

$$
A_{P, U}^{\phi} \phi_{P}=A_{N, U}^{\phi} \phi_{N}+A_{S, U}^{\phi} \phi_{S}+B_{U}^{\phi}
$$

where 


\section{TABLE I}

$$
\begin{aligned}
& \text { Diffusion Coefficients and Source Terms } \\
& \left(S^{\phi} \frac{\mathrm{r}}{\mathrm{r}} \Delta \mathrm{r}=\mathrm{S}_{1}^{\phi} \phi_{\mathrm{p}}+\mathrm{S}_{2}^{\phi}\right)
\end{aligned}
$$

$\phi$

1

$u$

k

$\varepsilon$

$$
\Gamma^{\phi}
$$

0

$\mu$

$$
\mu / \sigma_{k}
$$

$$
\mu / \sigma_{\varepsilon}
$$

$\mathrm{S}_{2}^{\phi}$ 0

$-\frac{\partial P}{\partial x}$

G

$C_{1} C_{\mu} \mathrm{Gok} / \mu$

IN THIS TABLE CERTAIN QUANTITIES AND CONSTANTS ARE DEFINED AS FOLLOWS:

$$
\begin{gathered}
\mu=\frac{C_{\mu} \rho k^{2}}{\varepsilon}+\mu_{\ell} \\
G=\mu\left\{2\left[\left(\frac{\partial V}{\partial r}\right)^{2}+\left(\frac{V}{r}\right)^{2}\right\}+\left(\frac{\partial U}{\partial r}\right)^{2}\right\}
\end{gathered}
$$

$$
\mathrm{C}_{\mu}
$$

$C_{D}$

$C_{1}$

$\mathrm{C}_{2}$

$q_{k}$

$\sigma$

0.09

1.0

1.44

1.92

$1.0 \quad 1.2174$ 


$$
\begin{aligned}
A_{P, U}^{\phi}=A_{N, U}^{\phi}+A_{S, U}^{\phi}-\bar{r} \Delta r \Delta S_{l}^{\phi} \\
+(\bar{r} \Delta r \rho u)_{P, U}-\left(r_{n} \Delta x \rho v\right)_{n, U}+\left(r_{S} \Delta x \rho\right)_{S, U} \\
A_{N, U}^{\phi}=\frac{\left(r_{n} \Delta x \Gamma^{\phi}\right)_{n, U}}{\Delta Y_{n}}-\frac{\left(r_{n} \Delta x \rho v\right)_{n, U}}{2} \\
A_{S, U}^{\phi}=\frac{\left(r_{S} \Delta x \Gamma\right)_{s, U}}{\Delta Y Y_{S}}-\frac{\left(r_{s} \Delta x \rho v\right)_{s, U}}{2} \\
B_{U}^{\phi}=(\bar{r} \Delta r \rho u)_{P, U} \phi_{P, U}+\bar{r} \Delta r \Delta x S_{2}^{\phi}
\end{aligned}
$$

The symbol $U$ used as the second subscript on the coefficients of $\phi$ in the above equations satisfies the fact that coefficients are to be evaluated using upstream (U) conditions which of course is necessitated by the parabolic nature of the calculations. This general equation takes on the following specific form for $\phi=1$, $u$, $k$ and $\varepsilon$ where $\Gamma^{\phi}, S_{1}^{\phi}$, and $S_{2}^{\phi}$ are defined in Table $I_{\text {. }}$

Let $\phi=1$, then

$$
(\bar{r} \Delta r \rho u)_{P, D}-(\bar{r} \Delta r \rho u)_{P, U}+\left(r_{n} \Delta x \rho v\right)_{n, U}+\left(r_{s} \Delta x \rho v\right)_{s, U}
$$

Let $\phi=u$, then

$$
u_{P}=\frac{A_{N, U}^{u}}{A_{P, U}^{u}} u_{N}+\frac{A_{S, U}^{u}}{A_{P, U}^{u}} u_{S}+\frac{B_{U}^{u}}{A_{P, U}^{u}}+\frac{D_{U}^{u}}{A_{P, U}^{u}}\left(\frac{\partial \bar{P}}{\partial x}\right)
$$


Let $\phi=k$, then

$$
k_{P}=\frac{A_{N, U}^{k}}{A_{P, U}^{k}} k_{N}+\frac{A_{S, U}^{k}}{A_{P, U}^{k}} k_{S}+\frac{B_{U}^{k}}{A_{P, U}^{k}}
$$

Let $\phi=\varepsilon$, then

$$
\varepsilon_{P}=\frac{A_{N, U}^{\varepsilon}}{A_{P, U}^{\varepsilon}} \varepsilon_{N}+\frac{A_{S, U}^{\varepsilon}}{A_{P, U}^{\varepsilon}} \varepsilon_{S}+\frac{B_{U}^{\varepsilon}}{A_{P, U}^{\varepsilon}}
$$

The pressure term in the axial equations will be discussed in the boundary condition section (2.3). The $\phi$ coefficients and the $B_{U}^{\phi}$ terms of the discretization equations are based only on upstream influence as indicated by the $U$ subscript. The unknown $\phi$ 's in the discretization equations are to be solved at a downstream station by the tri-diagonal matrix (TDM) algorithm.

\subsection{Boundary Conditions}

In the previous section the finite difference equations were developed for the interior of the flow. This section describes spectal forms of these equations used on the boundary. Since the flow is axisymmetric, only the top half of the flow field is considered with the boundaries being the top wall and the centerline. The special forms for the axial velocity, turbulence kinetic energy, and turbulence dissipation rate will be presented collectively at the centerline and individually at the wall. The calculation of radial velocity will be discussed in the next section. 
At the centerline since the flow is axisymmetric the variables $u$, $k$, and $\varepsilon$ have a zero radial gradient. The coefficients $A_{S, U}^{u}, A_{S, U}^{k}$, and $A_{S, U}^{\varepsilon}$ belonging to the centerline discretization equations are set equal to zero to satisfy this boundary condition.

In preparation for considering the conditions to be applied along the combustor wall, it is Important to keep in mind that the axial velocity, turbulence kinetic energy, and turbulence dissipation rate equations apply only for high Reynolds numbers. Near the wall, however, the flow is dominated by the laminar viscosity (10). To provide appropriate modeling in the near wall region, $u$ and $k$ equations will be modified by a wall function and the $\varepsilon$ equation will force $\varepsilon$ to be a fixed value. These near wall equations will use the control volume shown in Figure 3 and the wall function defined in Appendix A. The coefficients $A_{N, D}^{u}, A_{N, D}^{k}$, and $A_{N, D}^{\varepsilon}$ assoclated with the $u, k$, and $\varepsilon$ discretization equations will be set equal to zero so that the influence of the wall is handled entirely by the source terms $s^{u}, s^{k}$, and $s^{\varepsilon}$. The source terms for each near wall equation supplement the source terms associated with these equations for an interfor control volume.

The source term needed to modify the axial velocity discretization equation at the near wall region follows (9).

$$
S^{U}=\int_{V} \tau_{w} d V
$$

where 


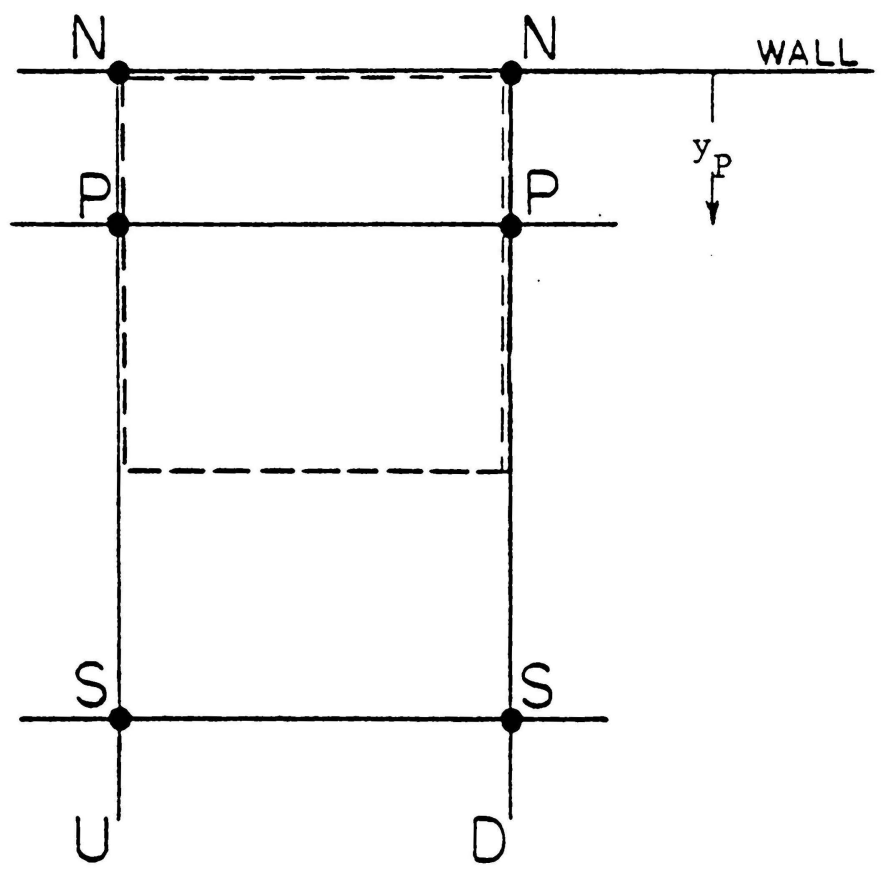

Fig. 3. Wall Parabolic Control Volume 


$$
\tau_{w}= \begin{cases}-\frac{\mu_{\ell}\left(u_{P}-u_{N}\right)}{y_{p}} & ; Y^{+}<11.63 \\ -\frac{\rho \mathrm{C}_{\mu}^{1 / 4_{k} 1 / 2}\left(u_{P}-u_{N}\right)}{\log _{e}\left(E Y^{+}\right)} & ; Y^{+}>11.63\end{cases}
$$

and

$$
\mathrm{Y}^{+}=\frac{y \sqrt{\frac{\tau}{\rho}}}{\nu} \approx \frac{Y \rho C_{\mu}^{1 / 4} k^{1 / 2}}{\mu_{\ell}}
$$

The source term necessary to modify the near wall turbulent kinetic energy equation follows (9)

$$
S^{k}=\int_{V}\left(G-C_{P} \rho \varepsilon\right) d V
$$

The calculations of this expression may be broken into

$$
\int_{V} G d V \approx \frac{\tau_{w}\left(u_{P}-u_{N}\right)}{y_{P}} d V
$$

and

$$
\int_{V} C_{P} \rho \varepsilon d V \approx \frac{-C_{P} \rho C_{\mu}^{3 / 4}\left(k_{P, U}^{1 / 2} k_{P, D}-k_{N, U}^{3 / 2}\right) U^{+} d V}{y_{P}}
$$

$\tau_{w}$ is calculated from Equation 17 and $\mathrm{U}^{+}$is calculated from

$$
U^{+}= \begin{cases}Y^{+} & \text {for } Y^{+}<11.63 \\ \frac{1}{K} \log _{e}\left(\mathrm{EY}^{+}\right) & \text {for } \mathrm{Y}^{+}>11.63\end{cases}
$$


The value of $\varepsilon$, unlike value of $k$, reaches a much higher value near the wall than anywhere else (9). The control volume for $\varepsilon$ that extends up to the wall is very difficult to model due to "shear ignorance" (9) of $\varepsilon^{\prime} s$ near wall profile. To eliminate this problem $\varepsilon$ is given a fixed value which is not a function of $\mathrm{Y}^{+}(9)$. The infital sublayer allows $\varepsilon$ to be expressed as

$$
\varepsilon_{P}=\frac{c_{\mu}^{3 / 4} k_{P, U}^{3 / 2}}{k y_{P}}
$$

\subsection{Solution Technique}

The axial velocity can be obtained from the axial momentum equation if the axial pressure is known from Equation 13. The guessed pressure gradient used in this equation is obtalned from the upstream station. In general, the estimated flow rate based on the guessed pressure will not satisfy conservation of mass. The following equations developed in Appendix B will be used to correct pressure gradient and the axial velocity.

$$
\begin{aligned}
& \frac{\partial \bar{P}}{\partial x}=\left(\frac{\partial \bar{P}}{\partial x}\right)^{*}+\left(\frac{\partial \bar{P}}{\partial x}\right)^{\prime} \\
& u_{P}=u_{P}^{*}-\frac{D_{U}^{u}}{A_{P, U}^{u}}\left(\frac{\partial \bar{P}}{\partial x}\right)^{\prime}
\end{aligned}
$$




$$
\left(\frac{\partial \bar{P}}{\partial x}\right)^{\prime}=-\left[\frac{\sum_{i=1}^{j} \rho_{1} U_{1} r_{1} \Delta r_{1}-\sum_{i=1}^{j} \rho_{i} U_{1}^{*} r_{1} \Delta r_{1}}{\sum_{i=1}^{j} \rho_{1} D_{U_{i}}^{u} r_{1} \Delta r_{1}}\right]
$$

The * in the above equations represents axial velocity based on the previous pressure gradient. A numerical procedure for stabilizing the axial velocity calculation is presented in Appendix Dl.

The discussion of radial velocity delayed earlier will now be presented. Radial velocity is calculated from Equation 12. The boundary conditions for $v$ are zero at the wall and centerline. Since the radial grid is highly nonunfform, the control volumes vary in size. In order to reduce the numerical error of the calculations the radial velocity is calculated inward from the wall and outward from the centerline to the largest control volume. The radial velocity calculated by the conservation of mass is stabilized by a numerical procedure presented in Appendix D2.

The turbulence kinetic energy is calculated from Equation 14 and then the turbulence dissipation rate is calculated from Equation 15. From the value of $k$ and $\varepsilon$ the turbulent viscosity is calculated from Equation 5 in preparation for taking the next downstream step. 


\section{PIPE FLOW TEST CASE}

In order to check out the parabolic code, a simple test case was run. The test case consists of laminar flow of air in a clrcular pipe. This is a well known problem called Poiseuille flow (11) for which there is an exact solution.

\subsection{Problem Description}

The data used in this test case was:

$\begin{array}{ll}\text { Variable } & \frac{\text { Value }}{\text { Dynamic Viscosity }} \\ \text { Density } & .1711 \times 10 \mathrm{~N} \mathrm{~s} / \mathrm{m}^{2} \\ \text { Diameter } & 1.19 \mathrm{~kg} / \mathrm{m}^{3} \\ \text { Temperature } & 0.0964 \mathrm{~m} \\ u_{\max } & 293.0 \mathrm{~K}\end{array}$

This results in a Reynolds number of 2000 which, since this is a laminar calculation, is necessarily less than the commonly accepted critical Reynolds number for pipe flow of 2300 . The results of the analysis are represented by the following equations:

$$
\begin{gathered}
u=\frac{d P}{d x} \frac{1}{4 \mu_{\ell}}\left(r^{2}-R^{2}\right) \\
\tau=\mu_{\ell} \frac{d u}{d r}=\frac{d P}{d x} \frac{r}{2}
\end{gathered}
$$




$$
\frac{d P}{d x}=-\frac{u_{\max } 4 \mu_{\ell}}{R^{2}}
$$

At the inlet to the pipe the axial velocity profile given by Equation 27 was input along with the value of the pressure gradient given by Equation 29. The value of the radial velocity at the inlet was set equal to zero. From these calculations the axial velocity, the radial velocity, and the axial pressure gradient were calculated. The axial mesh spacing from the calculations was 0.005 m. The pipe was $0.6 \mathrm{~m}$ long.

\subsection{Results}

The intial velocity profile along with the veloctty profiles of every tenth station are plotted in Figure 4. The ordinate represents the radial grid spacing from the centerline to the wall. The abscissa Indicates the magnitude of the axial velocity which increases from 0.0 $\mathrm{m} / \mathrm{s}$ at the wall to $u_{\max }=0.30 \mathrm{~m} / \mathrm{s}$ at the centerline. There is no change In the axial velocity profile from the initial station to the final station just as would be expected from the exact solution. Therefore in Figure 4 only a single curve is plotted which in fact represents the initial velocity as well as the velocity profile at each of the 120 axial stations at which calculations were made.

Although not specifically shown here, the radial velocities remain effectively zero. The radial velocity is between 4 and 5 orders of magnitude less than $u_{\max }$ with the sign being alternately positive and negative with each successive step. 


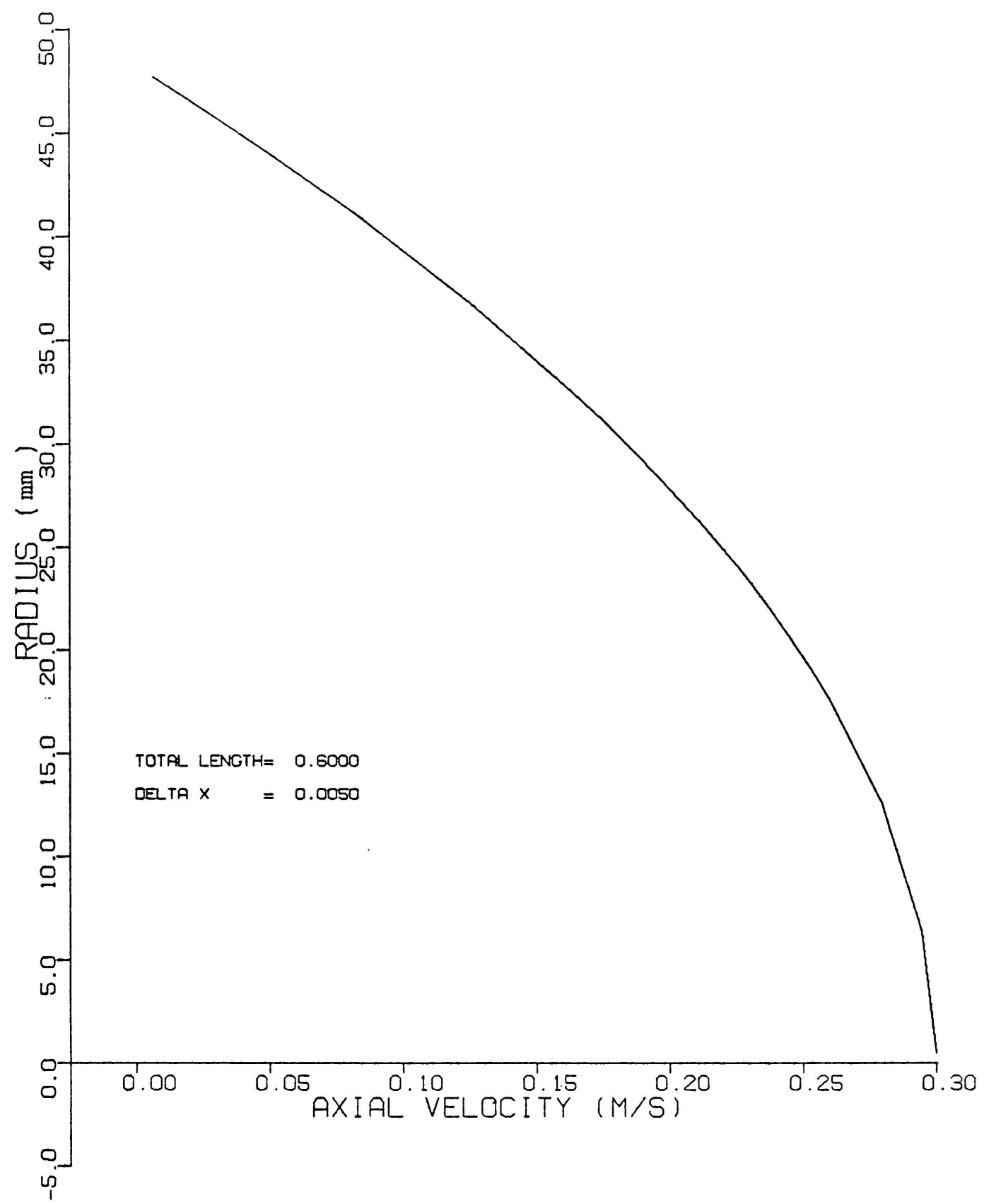

Fig. 4. Axial Velocity Profile Calculations for Fully Developed Pipe Flow 
of particular interest is the pressure gradient presented in Table II. The pressure gradient calculations are presented at the initial $x$ station and every following tenth axial station. The value of each axial pressure gradient is virtually the same as the exact solution $(-0.008825)$ 
TABLE II

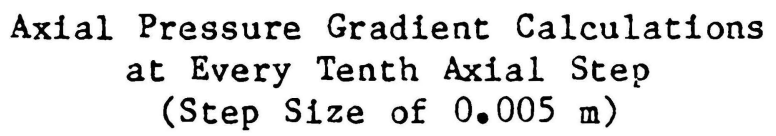

\begin{tabular}{|c|c|c|}
\hline Station & $x(m)$ & Axial Pressure Gradient \\
\hline 1 & .00 & -.008825 \\
\hline 10 & .05 & -.008866 \\
\hline 20 & .10 & -.008854 \\
\hline 30 & .15 & -.008849 \\
\hline 40 & .20 & -.008848 \\
\hline 50 & .25 & -.008845 \\
\hline 60 & .30 & -.008842 \\
\hline 70 & .35 & -.008843 \\
\hline 80 & .40 & -.008843 \\
\hline 90 & .45 & -.008841 \\
\hline 100 & .50 & -.008840 \\
\hline 110 & .55 & -.008841 \\
\hline 120 & .60 & -.008841 \\
\hline
\end{tabular}




\section{INTERFACING}

The ultimate utilization of the parabolic code is in conjunction with an elliptic code. This necessitates a procedure for transitioning from the elliptic calculations to the parabolic calculations. The radial velocity, turbulence kinetic energy, turbulence dissipation rate, and the dynamic viscosity are calculated and stored in exactly the same location in the elliptic and parabolic codes. Therefore, no special procedure will be needed to transfer these dependent variables from the elliptic code as initial conditions for the parabolic code. A special procedure, however, will be needed in conjunction with the axial velocity. The axial velocity calculated upstream and downstream of the parabolic starting station from the elliptic code is averaged to obtain a value of axial velocity at the station where the parabolic calculations begin:

$$
u_{P, U}=\frac{u_{P}+u_{E}}{2} .
$$

A special procedure is also necessary to obtain the initial pressure and axial pressure gradient for the parabolic code. Since the elliptic radial pressure is approximately the same at each radial location, the pressure at node 12 (a point approximately halfway between the wall and the centerline) is taken as the starting pressure for the parabolic code. The axial pressure gradient is also obtained at node 12 by subtracting the pressure of the elliptic station lmnediately following the elliptic/parabolic interface from the 
pressure at the interface station and dividing this difference divided by the axial distance between these two stations.

In order to take full advantage of the hybrid method, the parabolic code should start as soon after the reattachment point as possible, thereby saving the most computational time and machine storage. Although such a procedure is desirable from the standpoint of saving computational time and reducing storage, the parabolic calculations starting too close to the recirculating region run the danger of decreasing accuracy since the parabolizing assumptions neglect the streamwise diffusion terms. Consequently an interface location must be sought which represents a desirable tradeoff between accuracy, computational time, and machine storage.

One of the original purposes for carrying out this work was to perform numerical experimentation to provide some guidelines for selecting an appropriate starting location for the parabolic calculations. However, the expanding grid used by the elliptic code results in such a coarse mesh spacing downsteam of the reattachment point that the elliptic calculations of the flow field in this region were poor. These results made it impossible to carry out a systematic Investigation of the proper location for the interface of the two codes. More will be sald on this in the next section. 


\section{RAMJET COMBUSTOR CALCULATIONS}

\subsection{Geometry and Test Conditions}

In order to test the accuracy of the hybrid calculations the flow In the dump combustor was calculated and compared with the flow resulting from an elliptic calculation and with experimental data. The dump combustor is shown in Figure 5. The value of $R_{1}$ was chosen to be $0.0482 \mathrm{~m}$ and the value of $R_{2}$ was chosen to be $0.0254 \mathrm{~m}$. The Inlet velocity $\left(U_{0}\right)$ prior to opposition was taken to be $25.1 \mathrm{~m} / \mathrm{s}$. Alr is the working fluid. The diameter, density, dynamic viscosity, and temperature for the calculations are the same as that used in the pipe flow test case (Section 3).

\subsection{Calculations}

In a production-type application of the hybrid method, the parabolic calculations would be initiated by a partial solution of the ramjet combustor with the elliptic code. For the calculations described here, however, the entire dump combustor geometry was solved with STARPIC to provide the inftial conditions for the parabolic calculations. The elliptic code predicts reattachment just after station $35(x=0.18 \mathrm{~m})$ on an $11 \%$ expanding grid which extends from $x$ $=0.0 \mathrm{~m}$ (station 1) at the entrance to $\mathrm{x}=0.67 \mathrm{~m}$ (station 48) at the exit. The farthest upstream station at which the parabolic calculations could begin was at station 35. This section will describe these calculations and compare the results of the parabolic calculations carried out to station 40 and 43 with the elliptic 


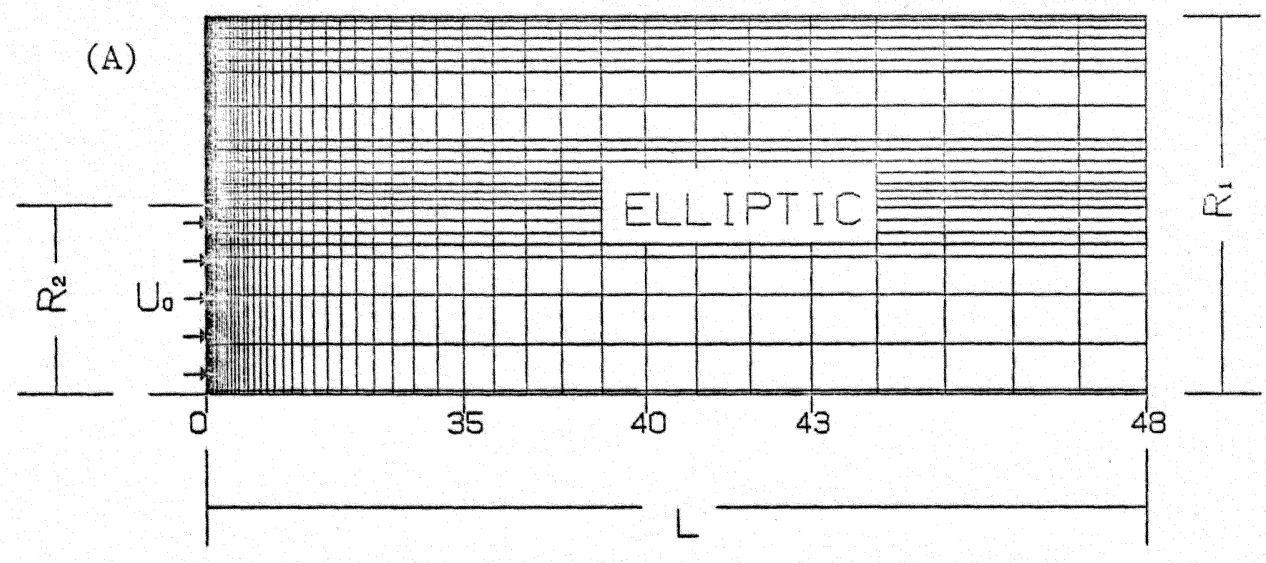

(B)

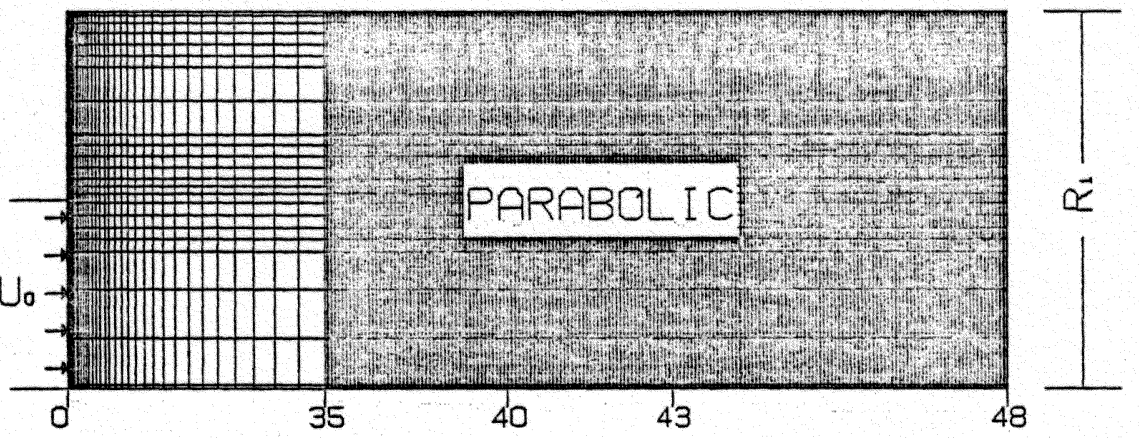

\begin{tabular}{|c|c|c|}
\hline STATION & $x(m)$ & $\frac{x}{R_{2}}$ \\
\hline 35 & 0.18414 & 7.25 \\
\hline 40 & 0.30481 & 12.00 \\
\hline 43 & 0.43183 & 17.00 \\
\hline 48 & 0.67249 & 26.48 \\
\hline
\end{tabular}

Fig. 5. Dump Combustor Axial Grid Spacing Used for the Elliptic Method (A) and the Hybrid Method (B) 
calculations and experimental data. The parabolic calculations use a constant $x$ grid mesh spacing of $0.001 \mathrm{~m}$.

\subsection{Results}

The solution of the equations presented in Section 4 allows the calculation of the axial and radial velocities, the local static pressure, the turbulence kinetic energy, and turbulence dissipation rate at each point in the flow field. In the comparison presented here, however, only the axlal velocity, and turbulent intensity are shown since these are the only quantities for which experimental data was available. The experimental data was obtained from Craig et al. (12) and Stevenson et al. (13). The axial locations at which these data were taken correspond to station 40 and 43 in the present calculations (see Figure 5). The calculations at station 40 are shown in Figures 6 and 7. The axial velocity and turbulent intensity are nondimensionalized by the velocity fust before expansion $\left(U_{0}\right)$ and plotted as the abscissa in Figures 6 and 7 respectively. The ordinate of both figures represents the radius nondimensionalized by the radius of the pipe before expansion $\left(R_{2}\right)$.

The results in Figure 6 indicate as expected an increase in velocity as the centerline is approached. Clearly the presence of the recirculating zone is strongly felt at this station and as expected is far from a fully developed turbulent profile. It is interesting that the parabolic calculations are in much better agreement with the experimental data than the elliptic calculations. The result is quite encouraging. There is, however, a disappointing nature to this 


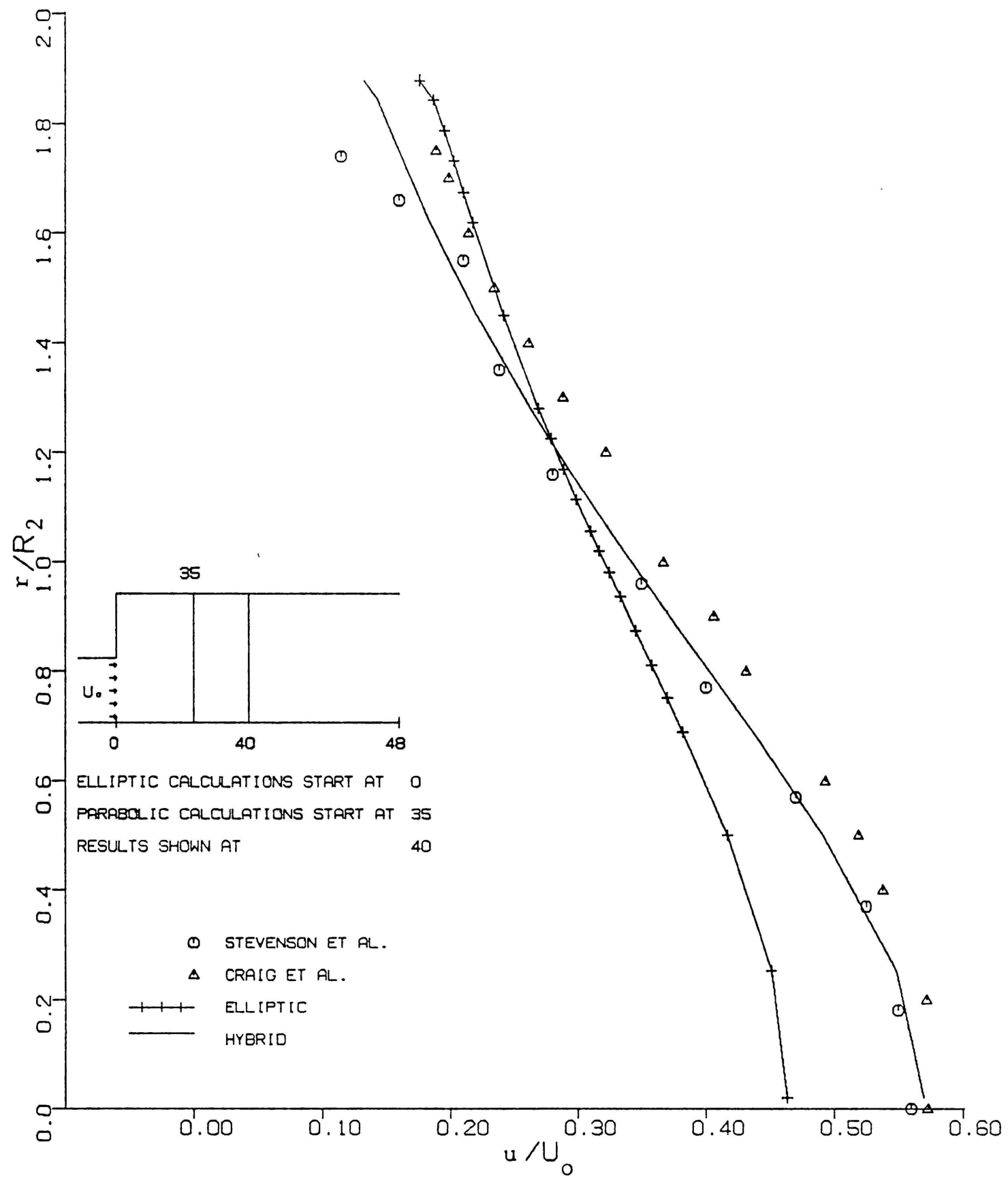

Fig. 6. Axial Velocity Results for the Hybrid Method, Elliptic Method, and Experimental Data at Station 40 


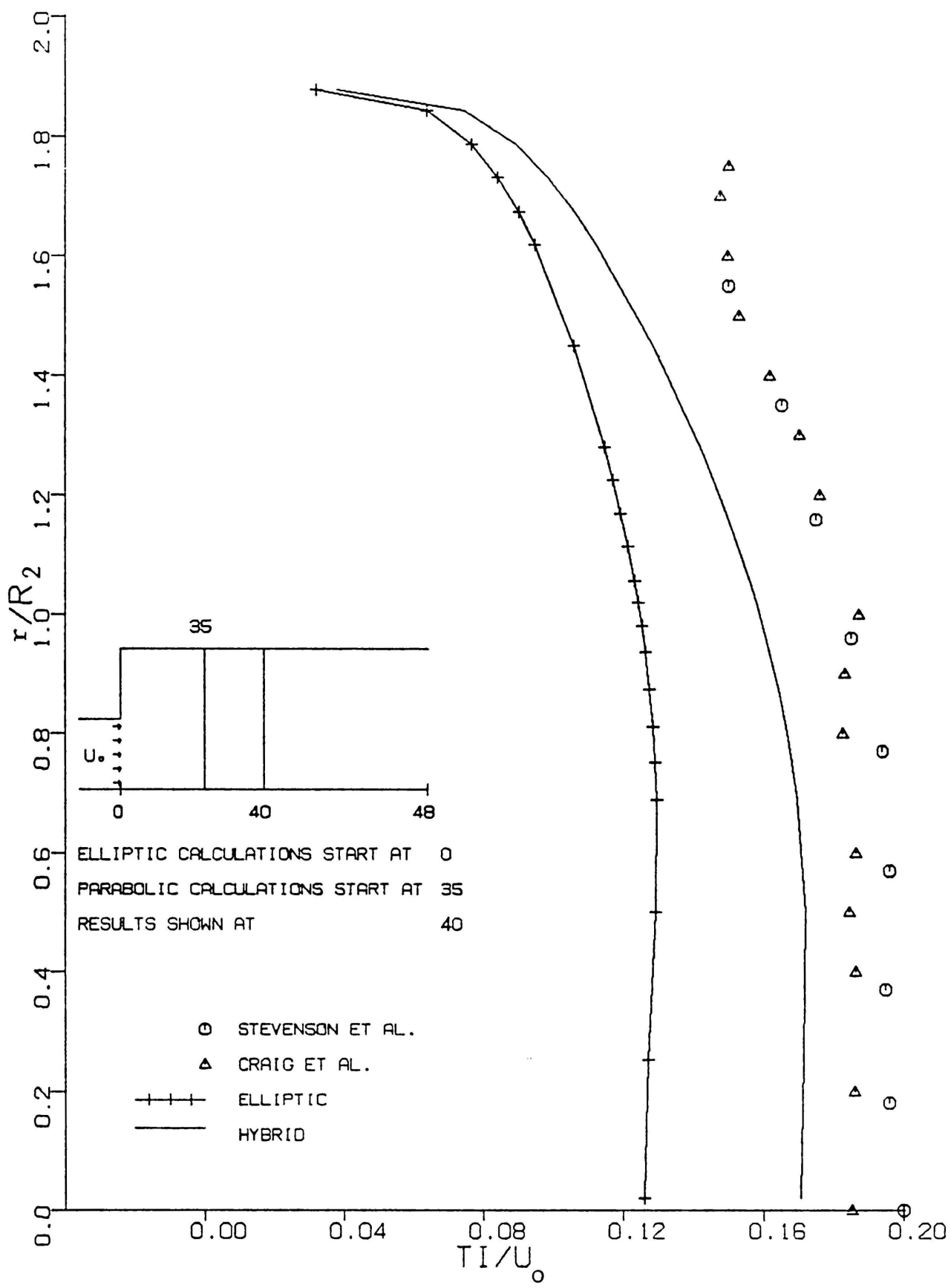

Fig. 7. Turbulent Intensity Results for the Hybrid Method, Elliptic Method, and Experimental Data, at Station 40 
discovery. It was initially planned to use station 40 to begin a second set of parabolized calculations. The poor agreement between the elliptic results and the experimental results at station 40 clearly point out the futility of such calculations. Therefore parabolic calculations beginning at station 35 will be the only calculations shown in this thesis. The excellent performance of the parabolic calculations at station 40 compared with the elliptic calculations requires an explanation. Since the elliptic code used the full Navier-Stokes equations and the parabolic code used an abridged form of the Navier-Stokes equations, how is it that the parabolic code out performs the elliptic code? This poor prediction of the flow fleld from the elliptic code is blamed on the highly expanded elliptic grid. In Figure 5A $72.9 \%$ of the nodes occur in the first $28.3 \%$ of the combustor. This high resolution allows an accurate description of the flow field to be obtained at station 35. Between station 35 and 48 , however, the remaining $71.7 \%$ of the combustor has only $27.1 \%$ of the nodes. This highly expanding $x$ grid is blamed for the severe loss of accuracy by the elliptic code in predicting the flow field around station 40. In contrast, the success of the parabolic calculations is attributed to the very high axial grid resolution of the calculations.

In Figure 7 the results of the turbulent intensity calculations are shown. Here even though the agreement of the parabolic calculations of experimental data is not as good as the case of the axial velocity they still agree much better with the experiments than do the elliptic calculations. The poor agreement of the elliptic 
calculations with the experimental turbulent intensity again points out the futility of choosing station 40 to begin the parabolic calculations.

Figures 8 and 9 are similar to Figures 6 and 7 , however, the calculations and experimental data are compared at station 43 rather than at station 40. The axlal velocity and turbulent intensity are shown on the same axes and with the same nondimensional parameters as In Figures 6 and 7. By the time this station is reached the distortions of the flow produced by the reclrculating zone are largely diffused and dissipated. The resulting flow calculations closely resemble turbulent pipe flow. Although from Figure 8 it is difficult to judge whether the better performer is the elliptic or the hybrid calculation, in Figure 9 it is quite clear that the hybrid results are superior in terms of predicting the turbulent intensity. The results which we have just seen are certainly gratifying from the standpoint of accuracy.

Given the inability of the elliptic calculation at station 40 and 43 to adequately predict the flow field one may be justifiably suspicious of the accuracy of the elliptic calculations at station 35 where the parabolic calculations began. An examination of Figure 10 should alleviate this concern. Figure 10, unlike Figure 9, shows only the turbulent intensity on the centerline. As previously indicated at stations 40 and 43 the agreement is poor, however, at station 35 where the parabolic calculations begin the agreement of the experimental data with the elliptic calculations is excellent. 


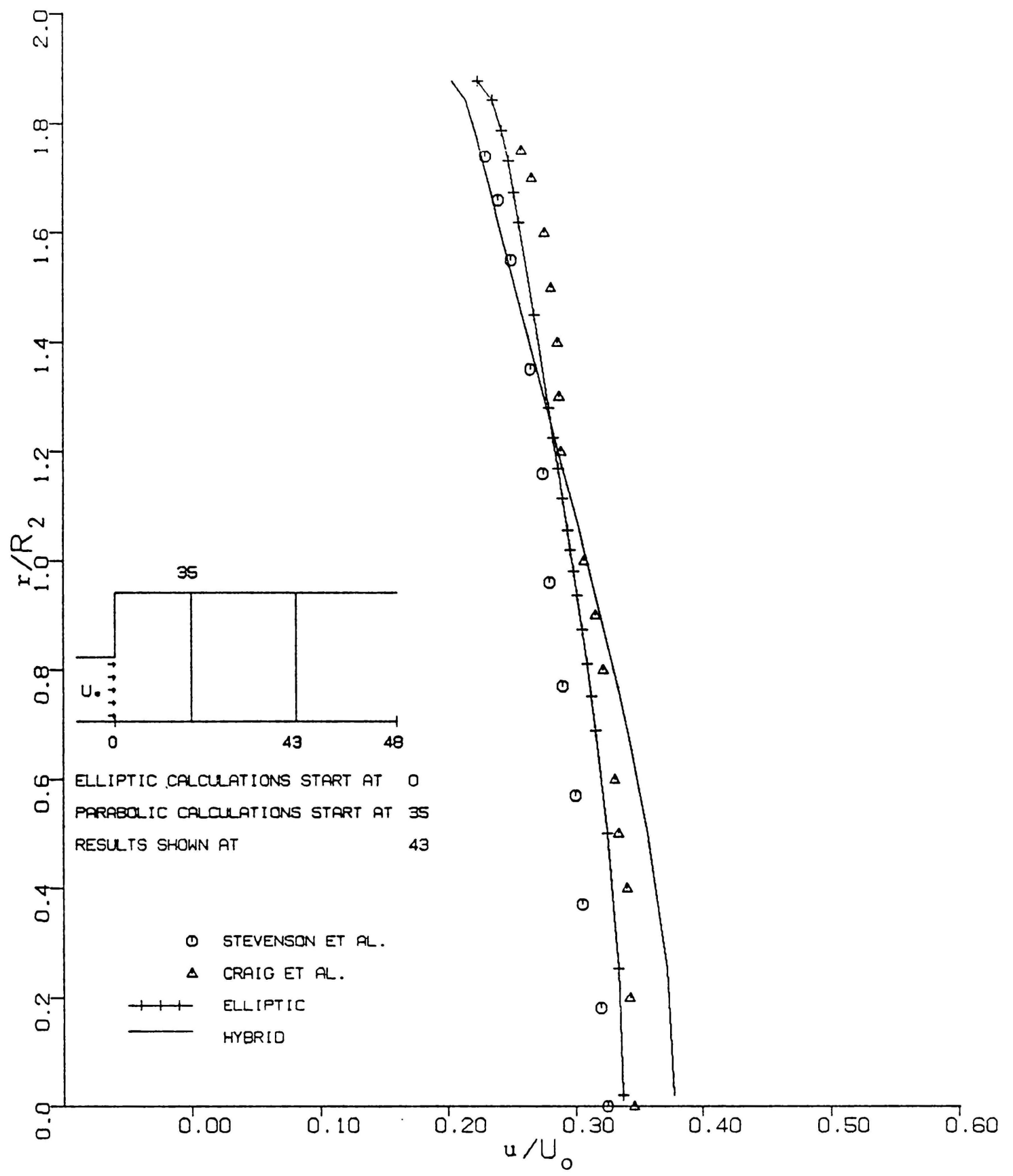

Fig. 8. Axial Velocity Results for the Hybrid Method, Elliptic Method, and Experimental Data at Station 43 


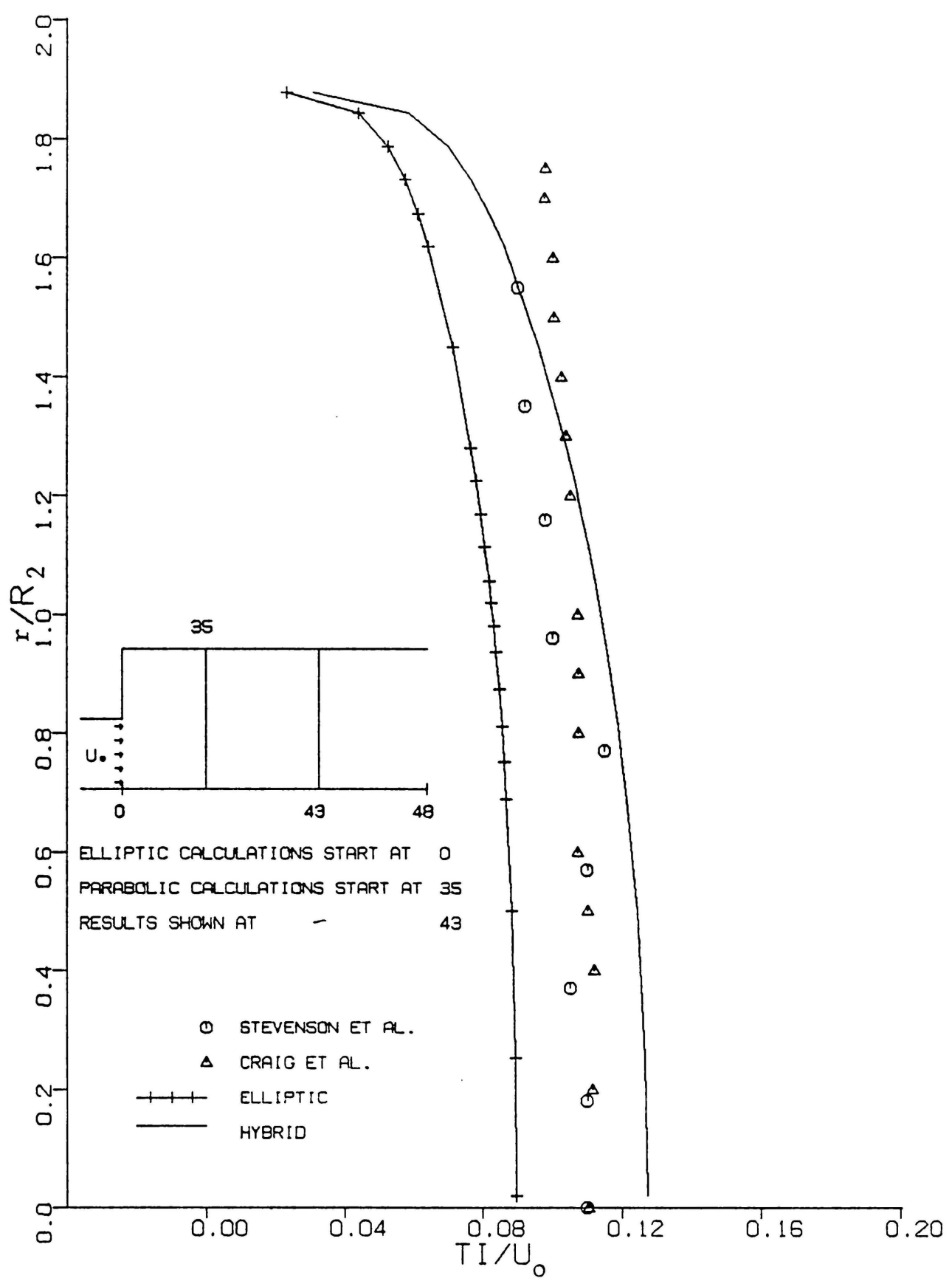

Fig. 9. Turbulent Intensity Results for the Hybrid Method, Elliptic Method, and Experimental Data at Station 43 


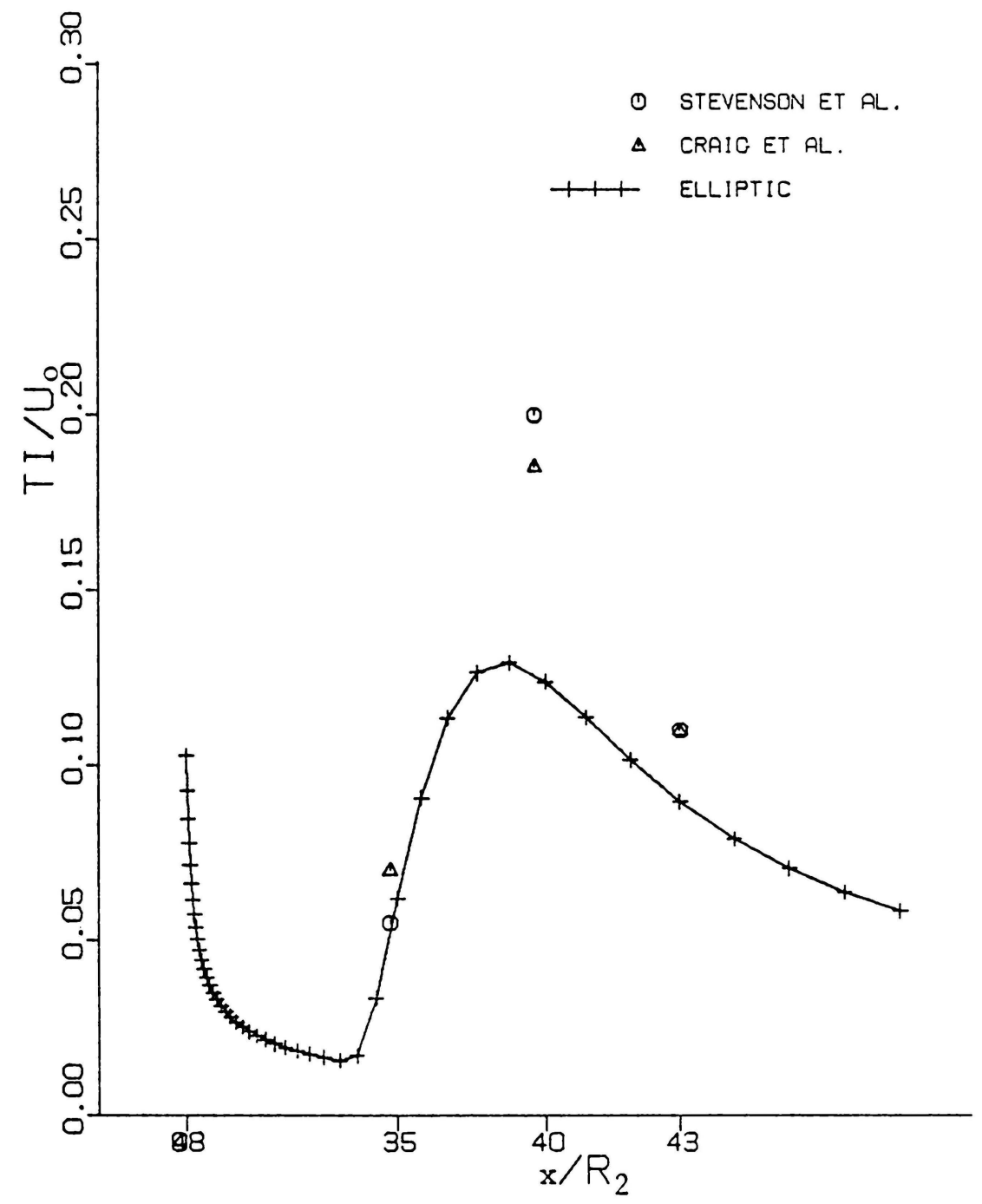

Fig. 10. Centerline Turbulent Intensity for Elliptic Calculations and Experimental Data at Each $\mathrm{x}$ Station 
Although no experimental data is available it is nevertheless interesting to look at the calculations of radial velocity. The results are shown in Figure 11 where the radial velocity profile is plotted as a function of radius at station 35 and station 43. In addition, calculations at every twentleth station are plotted between station 35 and 43. The results are reasonable. As station 43 is approached the radial velocity continually decreases. This is to be expected since the region of fully-developed pipe flow is being approached. Another method for calculating the radial velocity can be found in the Appendix. It uses the radial momentum (Navier-Stokes) equation. This method is discussed in Appendix $C$ with some stabilizing numerical procedure developed in Appendix D3.

\subsection{Computational Savings}

The original motivation for the hybrid method was to save computational time and machine storage but still maintain an acceptable degree of accuracy. The amount of computational time saved will be considered first.

Let us consider the requirements for the hybrid calculations first. The elliptic code which must be run to provide initial conditions to the parabolic calculations was run on the grid shown in Figure 5A. This required 3.88 minutes. The parabolic calculations required 0.17 minutes. The total time for the hybrid method was accordingly 4.05 minutes.

Let us now turn to the time required to make a similiar elliptic calculation. In order to present a fair comparison tt is necessary to 


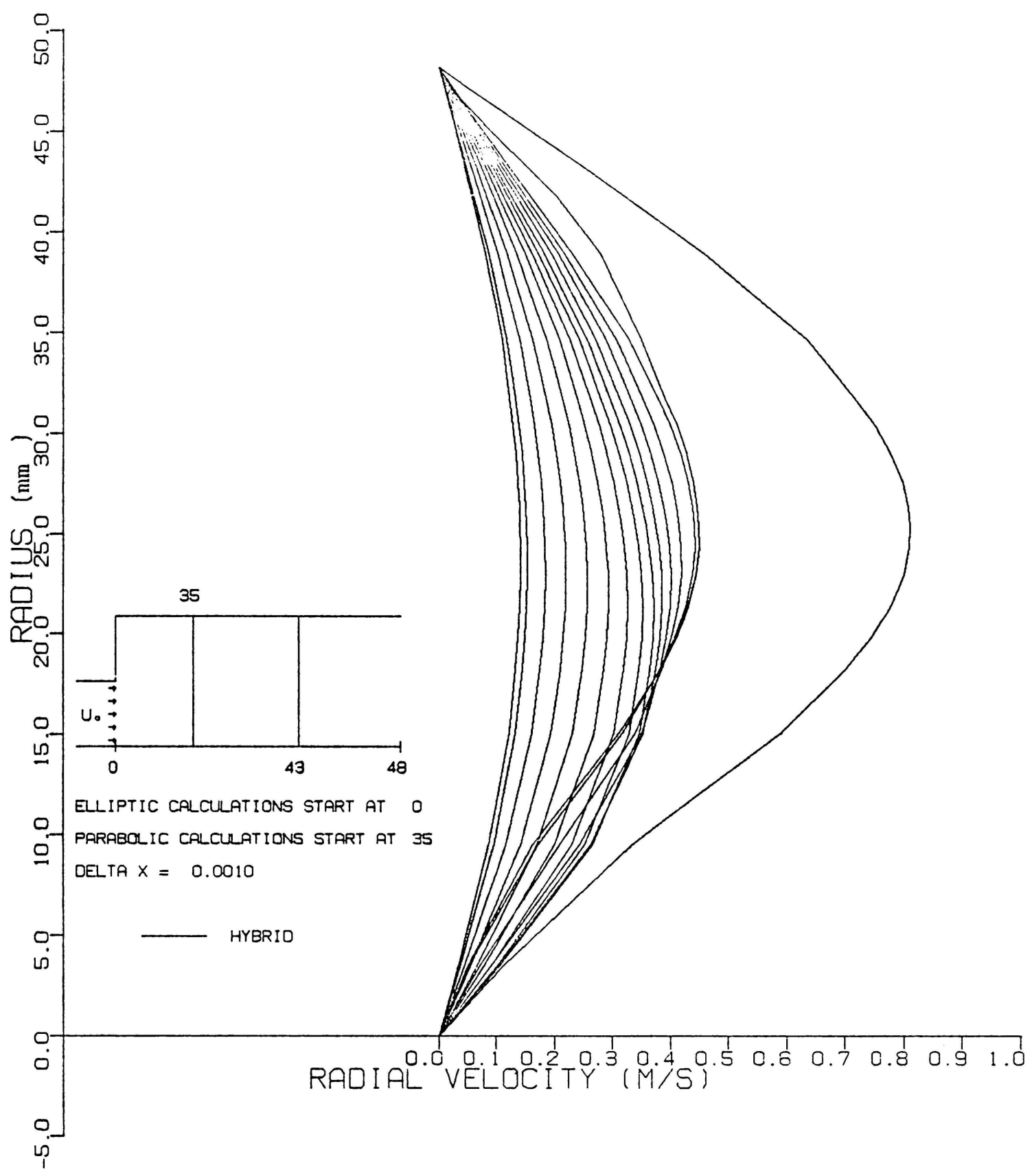

Fig. 11. Radial Velocity Plotted at Every Twentieth $\Delta \mathbf{x}$ From Station 35 to Station 43 by the Hybrid Method with a Step Size of $0.001 \mathrm{~m}$ 
run our elliptic calculations on the grid shown in Figure 5B. This in fact was never done. Therefore an estimate for the required computational time was made. The required computational time will be calculated by multiplying the time required to solve the flow field elliptically using the grid shown in Figure 5A (3.88 minutes) by the ratio of the number of axtal stations used in the hybrid method to the number of axial stations used in Figure 5A (523/48). The resulting estimate for the elliptic calculations on the same grid as that used by the hybrid method is 42.3 minutes. This estimate of computer time is admittedly conservative because it is a well known characteristic of iterative schemes that the computational time does not increas at a linear rate with mesh points but rather increases with the number of mesh points squared and in some cases cubed. As can be clearly seen by comparing the estimated 42.3 minutes required by the elliptic method to the 4.05 minutes required by the hybrid method there 1s at least an order of magnitude savings in computational time by using the hybrid method.

Let us now consider the machine storage saved by using the hybrid method Instead of the elliptic method. This is accomplished by first calculating the machine storage of the hybrid method. The required storage is that of the elliptic code (needed as inftial conditions for the parabolic calculations) and the parabolic calculations themselves. The machine storage used by the elliptic code is obtained from multiplying the 5 dependent variables ( $u, v, p, k$, and $\varepsilon$ ) by the radial locations (24) and the 48 axial planes. The elliptic code therefore requires 5752 storage locations. The machine storage needed 
for the parabolic code is determined by multiplying the 4 dependent variables ( $u, v, k$, and $\varepsilon$ ) by the radial locations (24) and the (only) 2 axial stations (an upstream and a downstream station). This results In 192 storage locations. Therefore, a total of 5952 machine storage locations are needed for the hybrid method.

To compare with the machine storage calculated for the hybrid method the same grid system will be used to obtain the machine storage needed to calculate the flow fleld with the totally elliptic method. An estimate of the number of storage locations is obtained by multiplying the 5 dependent variables (u, v, p, k, and $\varepsilon$ ) by the 24 radial locations and the 523 axial stations used in the hybrid mesh shown in Figure 5B. This multiplication shows a need for 62,760 machine storage locations using the totally elliptic method. A comparison of the 5952 machine storage locations needed for the hybrid calculations shows over an order of magnitude savings in the machine storage of the hybrid method. 


\section{CONCLUSIONS AND RECOMMENDATIONS}

The accuracy at which the parabolic code predicted the flow field is very satisfying. Unfortunately, a detailed study of the optimum location of the interfacing point could not be carried out; however, a satisfactory calculation was made. Savings in computational time and machine storage of more than one order of magnitude was achieved with no precelvable decrease in the accuracy of the calculations compared with elliptic calculations made with the same axial resolution.

By way of a recommendation I feel that the comparisons just reported should be repeated with adjustments made to the elliptic code to reduce the mesh spacing downstream of the reattachment point. This would allow the optimal starting location of the parabolic code to be found. The parabolic code should also be extended to handle compressible and reacting flows. 
1. Gosman, A. D. E. E. Khalil, and J. H. Whitelaw,"The Calculations of Two-Dimensional Turbulent Recirculating Flows," Imperial College of Science and Technology, London, 1978.

2. Lilley, D. G., and D. L. Rhode, "A Computer Code for Swirling Turbulent Axisymmetric Recirculating Flows in Practical Isothermal Combustor Geometries," NASA CR-3442, February 1982, pp. 6-30.

3. Novick, A. S., G. A. Miles, and D. G. Lilley, "Numerical Simulation of Combustor Flowfields: A Primitive Variable Design Capability," J. Energy, Vol• 3, No. 2, March-Apr11 1979.

4. Syed, S. A•, and G. J. Sturgess, "Validation Studies of Turbulence and Combustion Models for Aircraft Gas Turbine Combustors," Pratt and Whitney Alrcraft Division, Connecticut, November 1980.

5. Patankar, S. V., and D. B. Spalding, "A Calculation Procedure for Heat, Mass and Momentum Transfer in Three-Dimensional Parabolic Flows," Int. J. Heat Mass Transfer, Vol. 15, 1972, pp. 1787-1806.

6. Patankar, S. V., and D. B. Spalding, Heat and Mass Transfer in Boundary Layers, Intertext Books, London, 1970.

7. Spalding, D. B., Genmix: A General Compucer Program for TwoDimensional Parabolic Phenomena, Pergamon Press, London, 1977.

8. Patankar, S. V., Numerical Heat Transfer and Fluid Flow, McGrawHil1 Book Company, 1980.

9. Gosman, A. D., and F. J. K. Ideriah, "TEACH-2E: A General Computer Program for Two-Dimensional, Turbulent, Recirculating Flows," Dept• of Mech. Eng•, Imperlal College, June 1976.

10. Launder, B. E., and D. B. Spalding, "The Numerical Calculation of Turbulent Flows," Comp. Mthds. Appl. Mech. Eng., Vol. 3, 1974 , Pp. 269-289.

11. Fox, R. W., and A. T. McDonald, Introduction to Fluid Mechanics, John Wiley and Sons, Inc., 1973, p• 325•

12. Craig, R. R•, A. S• Nejad, E. Y. Hahn, and K. G. Schwartzkopf, "A General Approach for Obtaining Unbiased LDV Data in Highly Turbulent Non-Reacting and Reacting Flows," AIAA-84-0366, January 1984. 
13. Stevenson, W. W., H. D. Thompson, and T. S. Luchik, "Laser Velocimeter Measurements and Analysis in Turbulent Flows with Combustion - PART I," School of Mechanical Engineering, Purdue University, September 1982.

14. Harlow, F. H•, and P. I. Nakayama, "Turbulence Transport Equations," Physles of Fluids, Vol. 10, No. 11, November 1967. 
APPENDIX A

The Wall Function Definition

In the near wall region the local Reynolds number $\mathrm{Y}^{+}$increases rapidly. In order to accurately model the wall shear stress in this region $1 t$ is necessary therefore to define a wall function. The development of the wall function is guided by the one-dimensional Couette flow analysis in the region close to the wall where the shear stress can be assumed to be a constant. The Navier-Stokes (axial momentum) equation can be reduced by these assumptions to the following very simple nondimensional form (9).

$$
\frac{\tau}{\tau_{W}}=\left(1+\frac{\mu}{\mu_{l}}\right) \frac{\mathrm{dU}^{+}}{\mathrm{dy}^{+}}
$$

where

$$
U^{+}=\frac{u}{\sqrt{\frac{\tau_{w}}{\rho}}}
$$

and

$$
Y^{+}=\frac{Y}{r} u_{\tau} ; u_{\tau}=\sqrt{\frac{\tau}{\rho}}
$$

The general wall functions based on $\mathrm{Y}^{+}$are broken into three regions:
a) Viscous sublayer $\left(0<\mathrm{Y}^{+}<5\right)$ where $\frac{\mu_{\ell}}{\mu} \gg 1$. 
b) Buffer zone $\left(5<\mathrm{Y}^{+}<30\right)$ where laminar viscosity and turbulent viscosity are of the same magnitude such that neither dominates the flow.

c) Outer sublayer $\left(30<\mathrm{y}^{+}<400\right)$ where $\frac{\mu_{\ell}}{\mu} \ll 1$.

The approach taken by STARPIC and adopted by the parabolic scheme is to eliminate the buffer zone by assuming the flow is purely viscous from the wall to $\mathrm{Y}^{+}<=11.63$. For $\mathrm{Y}^{+}>11.63$, the flow is assumed to be fully turbulent (2). $\mathrm{Y}^{+}$undergoes a step change between two wall functions (9). Equation Al may be integrated in these two regions to obtain a calculation of $\mathrm{U}^{+}$as a function of $\mathrm{Y}^{+}$.

$$
U^{+}=\left\{\begin{array}{lc}
\mathrm{Y}^{+}, & \text {if } \mathrm{Y}^{+}<11.63 \\
\frac{1}{\mathrm{~K}} \log _{\mathrm{e}}\left(\mathrm{EY}^{+}\right), & \text {if } \mathrm{Y}^{+}>11.63
\end{array}\right.
$$

The Integration constant, $E=9.793$, depends on shear stress in the viscous sublayer and pipe roughness. The von Karman constant $k=$ 0.4187 .

In the inertial region $\left(0<\mathrm{Y}^{+}<400\right)$ mean velocity is considered to be independent of $y$ so the time averaged convection and diffusion terms in the energy transport equations average to zero. The turbulence equations reduce to Production = Dissipation (14). This assumption in the inertial region formulates the basis for the near wall shear stress $\left(\tau_{w}\right)$ calculations as a function of turbulent kinetic energy. An isotropic viscosity assumption (2) further reduces the $k$ equation to 


$$
\tau_{I}=C_{\mu}^{1 / 2} \rho k
$$

where $\tau_{W} \approx \tau_{I}$ and the $\varepsilon$ equation to

$$
\sigma_{\varepsilon}=\frac{x}{\left(c_{2}-c_{1}\right) c_{\mu}^{1 / 2}}
$$




\section{APPENDIX B}

Correction for Axial Velocity and Pressure

A pressure correction equation is needed to correct the axial velocity and update the pressure gradient. Equation 2, rewritten below for conventence, is used to calculate the axial velocity with the correct pressure gradient.

$$
u_{P}=\frac{A_{N, U}^{u}}{A_{P, U}^{u}} u_{N}+\frac{A_{S, U}^{u}}{A_{P, U}^{u}} u_{S}+\frac{B_{U}^{u}}{A_{P, U}^{u}}+\frac{D_{U}^{u}}{A_{P, U}^{u}}\left(\frac{\partial \bar{P}}{\partial x}\right)
$$

However, the pressure gradient is unknown so a guessed pressure gradient will be used to obtain an estimate to the axial velocity as Indicated by the * in the following equation.

$$
u_{P}^{*}=\frac{A_{N, U}^{u}}{A_{P, U}^{u}}+u_{N}^{*} \frac{A_{S, U}^{u}}{A_{P, U}^{u}} u_{S}^{*}+\frac{B_{U}^{u}}{A_{P, U}^{u}}+\frac{D_{U}^{u}}{A_{P, U}^{u}}\left(\frac{\partial \bar{P}}{\partial x}\right)
$$

In general the axial velocity profile obtained from this guessed pressure field will not satisfy the following conservation of mass equation.

$$
\dot{m}=2 \pi \sum_{i=1}^{j} \rho_{1} u_{i} r_{1} \Delta r_{1}
$$

The difference between Equation B1 and Equation B2 would represent the error assoclated with calculating the axial velocity from the guessed pressure as shown by 


$$
u_{P}=u_{P}^{*}+\frac{A_{N, U}^{u}}{A_{P, U}^{u}}\left(u_{N}-u_{N}^{*}\right)+\frac{A_{N, U}^{u}}{A_{P, U}^{u}}\left(u_{S}-u_{S}^{*}\right)+\frac{D_{U}^{u}}{A_{P, U}^{u}}\left(\frac{\partial \bar{P}}{\partial x}\right)^{\prime}
$$

where

$$
\frac{\partial \bar{P}}{\partial x}=\left(\frac{\partial \bar{P}}{\partial x}\right)^{*}+\left(\frac{\partial \bar{P}}{\partial x}\right)^{\prime}
$$

For the above equation to function as a velocity correction equation 1t must be explicit, therefore, the second and third terms on the right are eliminated.

$$
u_{P}=u_{P}^{*}+\frac{D_{U}^{u}}{A_{P, U}^{u}}\left(\frac{\partial \bar{P}}{\partial x}\right)^{\prime}
$$

The explicit axial velocity correction equation shown above is substituted into Equation B3 to calculate the following pressure gradient correction.

$$
\left(\frac{\partial \overline{\bar{P}}}{\partial x}\right)^{\prime}=-\left[\frac{\sum_{i=1}^{j} \rho_{1} u_{1} r_{1} \Delta r_{1}-\sum_{1=1}^{j} \rho_{1} u_{1}^{*} r_{1} \Delta r_{1}}{\sum_{1=1}^{j} \rho_{1} D_{U_{1}}^{u} r_{1} \Delta r_{1}}\right]
$$

This equation gives the required $\left(\frac{\partial \bar{P}}{\partial x}\right)^{\prime}$ necessary to update the guessed pressure gradient $\left(\frac{\partial \bar{P}}{\partial x}\right)^{*}$ from Equation $B 5$ and to correct the axial velocity from Equation B6. 


\section{APPENDIX C}

\section{Radial Momentum Equation for $v$}

An alternative to the conservation of mass equation for the calculation of the radial veocity ( $v$ ) is the following radial momentum (Navier-Stokes) equation:

$$
\frac{1}{r}\left[\frac{\partial}{\partial x}(r \rho u v)+\frac{\partial}{\partial r}(r \rho v v)-\frac{\partial}{\partial r}\left(r \Gamma \frac{\partial v}{\partial v}\right)\right]=-\frac{\partial P}{\partial x}+s^{v} \cdot
$$

The radial momentum equation may be written in finite difference form and integrated over a control volume to produce the following discretization form:

$$
v_{P}=\frac{A_{N, U}^{v}}{A_{P, U}^{v}} v_{N}+\frac{A_{S, U}^{v}}{A_{P, U}^{v}} v_{S}+\frac{B_{U}^{v}}{A_{P, U}^{v}}+\frac{D_{U}^{v}}{A_{P, U}^{v}}\left(P_{P}-P_{S}\right)
$$

As in the calculation of $v$ from the conservation of mass the radial velocity calculations follow the axial velocity and pressure gradient calculations. Since the radial pressure field is unknown a guessed radial pressure field is substituted into Equation C2 to obtain an estimate for $v$ as shown below.

$$
v_{P}^{*}=\frac{A_{N, U}^{v}}{A_{P, U}^{v}} v_{N}^{*}+\frac{A_{S, U}^{v}}{A_{P, U}^{v}} v_{S}^{*}+\frac{B_{U}^{v}}{A_{P, U}^{v}}+\frac{D_{U}^{v}}{A_{P, U}^{v}}\left(P_{P}^{*}-P_{S}^{*}\right)
$$

The * indicates the calculated radial velocity is based on a guessed radial pressure field $\left(P^{*}\right)$. The radial velocity based on the $P^{*}$ will not satisfy in general the conservation of mass equation by producing 
a net gain or loss in mass. A procedure to annihilate this net mass is to create a pressure correction from

$$
P=P^{*}+P^{\prime}
$$

where $P^{\prime}$ is the pressure correction, $P$ is the guessed pressure, and $P$ 1s the actual pressure. The radial velocity correction equation will be derived by subtracting the actual radial velocity equation (Equation $\mathrm{C2}$ ) based on the actual radial pressure field from the radial velocity equation (Equation 63 ) based on the guessed radial pressure field. The following radial velocity correction

$$
v_{P}=v_{P}^{*}+\frac{D_{U}^{v}}{A_{P, U}^{v}}\left(P_{P}^{\prime}-P_{S}^{\prime}\right)+\frac{A_{N, U}^{v}}{A_{P, U}^{v}}\left(v_{N}-v_{N}^{*}\right)+\frac{A_{S, U}^{v}}{A_{P, U}^{v}}\left(v_{S}-v_{S}^{*}\right)
$$

1s reduced to

$$
v_{P}=v_{P}^{*}+\frac{D_{U}^{v}}{A_{P, U}^{v}}\left(P_{P}^{\prime}-P_{S}^{\prime}\right)
$$

by dropping the last two terms. The last two terms must be dropped in order to reduce Equation C5 from enplicit to explicit Equation C6. The above corrected radial velocity equation is substituted into the finite differenced conservation of mass equation (Equation 12) to develop the following $P^{\prime}$ correction equation

$$
P_{P}^{\prime}=\frac{A_{N, U}^{P}}{A_{P, U}^{P}} P_{N}^{\prime}+\frac{A_{S, U}^{P}}{A_{P, U}^{P}} P_{S}^{\prime}+B_{U}^{P}
$$


This equation is solved by a TDM procedure to calculate the $\mathrm{P}^{\prime}, \mathrm{s}$. With the $P^{\prime}, s$ known, the radial pressure may be updated from Equation $\mathrm{C} 4$ and the radial velocity may be corrected from Equation $\mathrm{C6}$.

The dump combustor calculations described in Section 5 were recalculated with the radial velocity obtained from the radial momentum equation instead of the conservation of mass equation. The radial velocity calculation were essentially the same as that calculated from the conservation of mass. 


\section{APPENDIX D}

\section{Some Stabilizing Numerical Procedures}

The calculations of axial velocity and radial velocity required some stabilizing numerical procedures suggested by Mrs. Joan Moore.

\section{Axial Momentum}

The calculation of axial velocity obtained from the solution technique section was found to oscillate about the correct axial velocity from one axial step to the next. The axial velocity oscillation was eliminated by dividing the pressure correction term in Equation 25 by 2 as shown below:

$$
u_{P}=u_{P}^{*}-\frac{D_{U}^{u}}{A_{P, U}^{u} 2}\left(\frac{\partial \bar{P}}{\partial x}\right)^{\prime}
$$

2. Continuity

1) When the expressions

$$
(\rho v)_{n}=\frac{(\rho, v)_{n, D}+(\rho v)_{n, U}}{2}
$$

and

$$
(\rho v)_{s}=\frac{(\rho v)_{s, D}+(\rho v)_{s, U}}{2}
$$

are substituted into the following finite differenced conservation of mass equation 


$$
\frac{1}{r}\left[\frac{r_{n}(\rho v)_{n}-r_{s}(\rho v)_{s}}{\Delta r} \approx+\left[\frac{(\rho u)_{P, D}-(\rho u)_{P, U}}{\Delta x} \approx=0\right.\right.
$$

and solved for $v_{D}$, a numerical instability results. This problem is eliminated by assuming $(\rho v)_{n}$ and $(\rho v)_{s}$ are located at downstream stations.

2. The radial velocity was obtained from a highly nonuniform radial grid by solving the conservation of mass equation for radial velocity from the wall to the centerline. At the centerline the numerical round off error was significant. The solution was to solve the conservation of mass equation for radial velocity by calculating inward from the wall and outward from the centerline to the largest control volume. The largest control volume absorbed the numerical error.

3. The radial velocity was found to oscillate about the correct radial velocity profile form one axial step to the next. This radial velocity oscillation was eliminated by:

$$
v_{D}=\frac{v_{U}+v_{D}}{2}
$$

3. Radial Momentum

1. A pressure correction equation needed to correct the radial velocity is obtained for each control volume. However, since a global pressure correction has already been obtained to correct 
the axial velocity and update the axial pressure a full set of pressure correction equations would be indeterminate. This is due to the presence of one too many pressure correction equations. To eliminate this problem the pressure correction associated with the largest control volume is set equal to zero.

2. The radial velocity was found to osclllate about the correct radial velocity at each axtal step. The correction is the same as that developed earlier in Equation D5. 


\section{The vita has been removed from the scanned document}

